\title{
Secondary Breast Augmentation: Managing Each Case
}

\author{
Javier de Benito $\cdot$ Kyrenia Sanchez
}

Received: 21 August 2009/Accepted: 5 October 2010/Published online: 17 April 2010

(C) The Author(s) 2010. This article is published with open access at Springerlink.com

\begin{abstract}
Breast augmentation is one of the most regularly performed interventions requiring reoperation in aesthetic surgery. For this reason, it involves a greater chance for complications. In this report, the authors aim to provide young plastic surgeons with guidelines based on their experience for responding to each of these complications, to explain the causes and ways of avoiding them, and to show how they can be treated when they occur.
\end{abstract}

Keywords Breast augmentation - Complications .

Capsuloplasty $\cdot$ Submuscular pocket $\cdot$ Subglandular pocket

Breast augmentation is one of the most frequently performed operations involving plastic surgery. For this reason, breast augmentation involves a greater chance for complications that require reoperation. The causes for complications include capsular contracture, high implants, descent of submammary fold, rippling, visibility and palpability of the implant, double submammary fold, synmasty, infection and extrusion of the implant, rupture of the implant, changes in volume, and changes that occur due to the patient's age.

\section{Capsular Contracture}

Capsular contracture is one of the most frequent complications of breast augmentation (Fig. 1). It is almost

J. de Benito $(\varangle) \cdot$ K. Sanchez

Instituto Javier de Benito, Instituto Universitario Dexeus, Avenida Gran Via de Charles III, 71-75, 08028 Barcelona, Spain e-mail: drdebenito@gmail.com always multifactorial, caused by contamination (talc from gloves, cotton particles from gauze, cellulose from the blister box envelope), microtrauma (continual friction between the textured surface shell and the tissue around the implant; Fig. 2), hematoma, and infection (bacterial contamination from the rupture of cysts and mammary ducts). The symptoms may vary from slight to grave according to the degrees of capsular contracture described by Becker [1].

At the Institute de Benito we have a capsular contracture rate of $4 \%$. Of the capsular contractures, $86 \%$ involve submuscular implants. The continuous rubbing action of the pectoral muscle on the rough surface produces microtrauma, microinflammation, thickening of the capsule, and contracture. For this reason, it is not advisable to massage the breast when the implant has a textured surface $[2,3]$.

To prevent capsular contracture, attempts should be made to avoid, among other things, dust contamination from gloves and gauze particles. Capsular contracture also can be reduced by traumatizing tissues as little as possible. Therefore, surgeons should avoid creating the "pocket" with manual techniques (e.g., undermining with a finger) and instead should perform the dissection of the pocket with an electrocoagulater using a Colorado needle.

Once the capsular contracture occurs, it should be treated as follows. If the implant is positioned in a subglandular pocket and the patient presents a sufficient envelope, a total capsulectomy can be performed, with a new implant fitted inside the same pocket.

For cases in which the implant is fitted into a subglandular pocket, but the patient has hardly any tissue that covers the implant or the implant is atrophied, total capsulectomy should be performed and a submuscular pocket created, with a new implant fitted into it. 


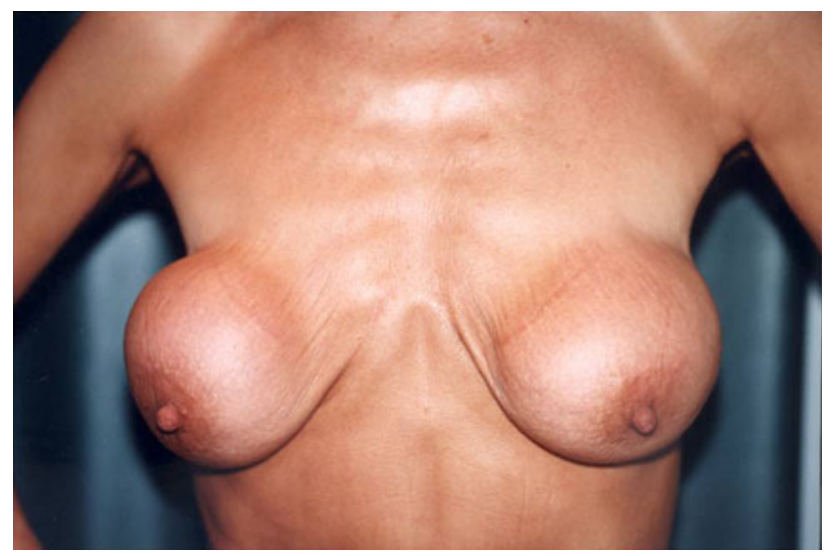

Fig. 1 Patient with capsular contracture (Baker 4)

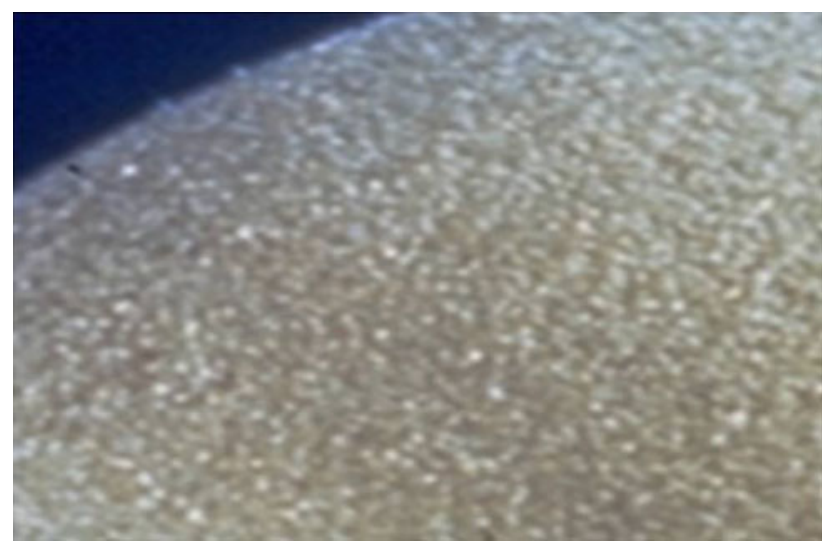

Fig. 2 Micropore textured surface

For cases in which the implant is fitted into a submuscular pocket, total or partial capsulectomy should be performed. The part of the capsule that remains adhering to the costal rib wall should be retained, with a new implant fitted into it.

Closed capsulectomy should be avoided because the immediate effect it creates is of short duration. It produces bleeding by breaking the tissues and can increase the capsula thickness as a result of hematoma, irregularities in palpation, rupture of the implant, and pain. On the other hand, with open capsulectomy, small amounts of silicone, talc, and other substances are eliminated. The tissues are left free for their appropriate distribution, thus avoiding irregularities or retractions [4-7].

\section{High Location of the Implant}

A high implant location can result from migration of the implant to the upper pole, especially when it is fitted via the armpit, or from the wrong location of the submammary fold. It also can result when the submammary fold should
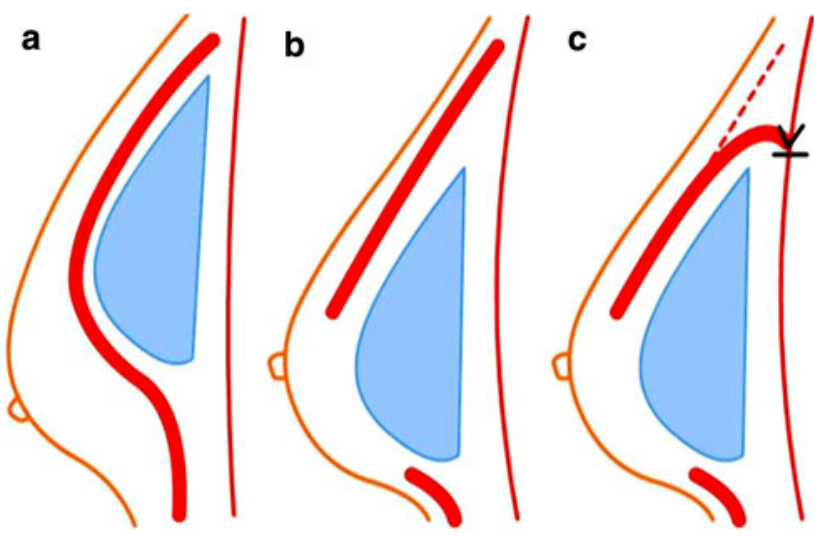

Fig. 3 a-c After lower pectoral muscle insertion and upper capsuloplasty, the patient is advised to avoid muscular activity for 3 weeks. A strip is fitted on the upper pole to prevent the implant from moving upward again $[3,8]$
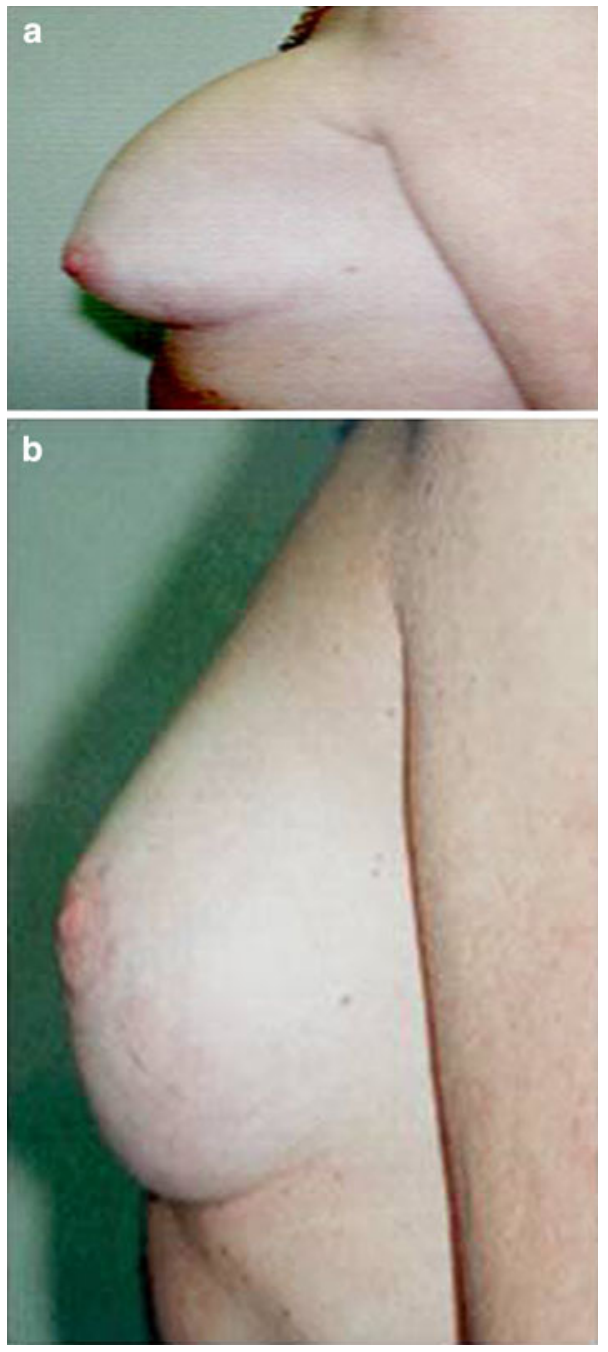

Fig. 4 a Implant located in the upper pole without pectoral release. b The pectoral muscle is released, and the implant is balanced with the nipple-areolar complex 
Fig. 5 a Submammary fold descent. b New submammary fold by the Hammock

technique. c Lateral half of the profile preoperatively. d Lateral half of the profile postoperatively. e Profile preoperatively. f Profile postoperatively
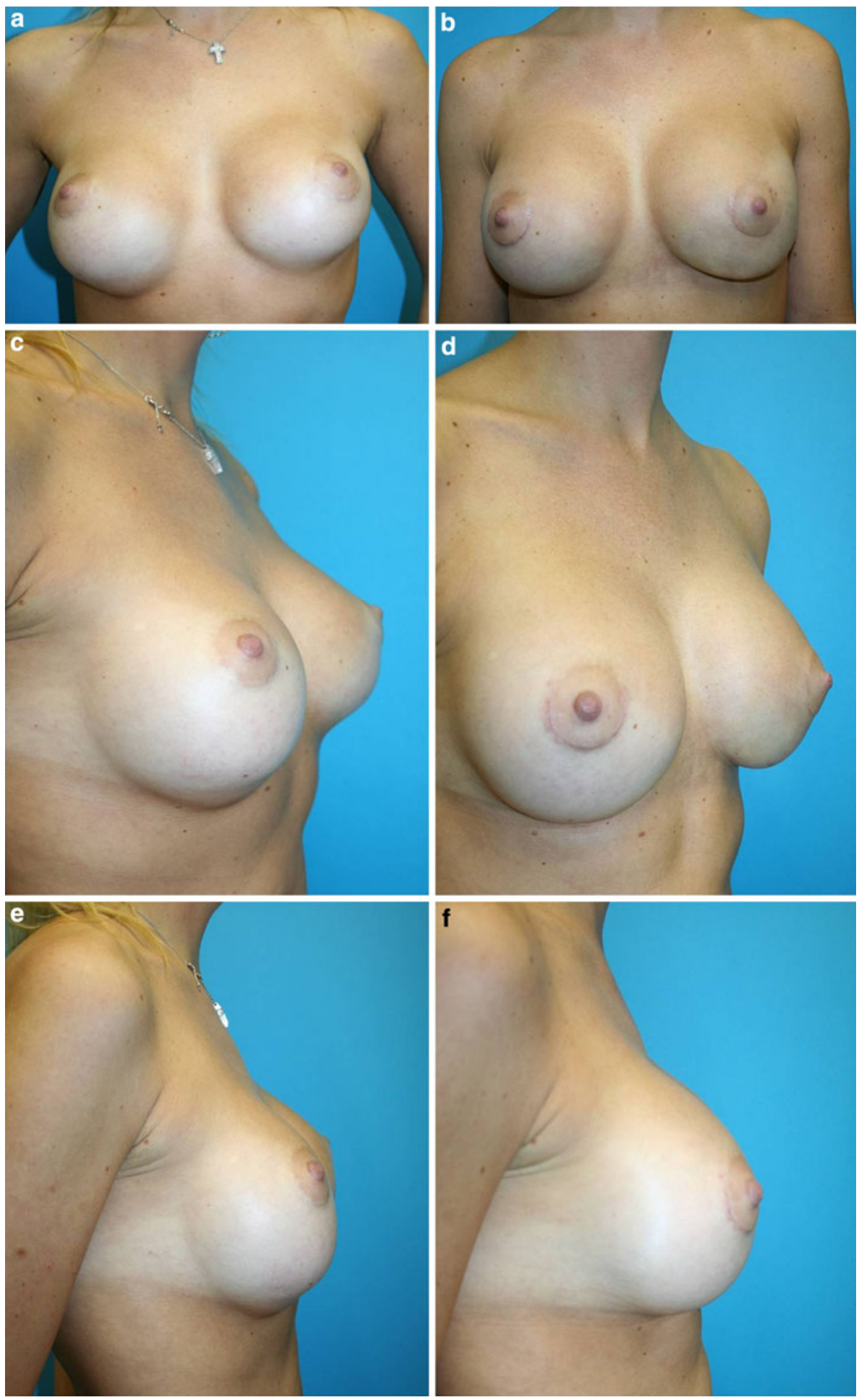

be lowered, but we do not release the low insertion of pectoral muscle. As a result, the implant remains high.

This problem can be solved as follows. If the implant is fitted subglandularly, the submammary fold should be lowered and a total capsulectomy performed. The pocket should be kept as it is, and an attempt should be made to center the implant in balance with the areola. If the implant is located submuscularly, a partial capsulectomy should be performed, leaving the part of the capsule adhering to the costal rib wall. A submuscular pocket should be performed with disinsertion of the lower portion of the pectoral muscle. An attempt should be made to fit the implant in balance with the nipple-areola complex, and an upper capsuloplasty should be performed (Fig. $3 \mathrm{a}-\mathrm{c}$ ) to avert the 
possibility of the implant becoming displaced upward (Fig. 4a and b).

\section{Descent of the Submammary Fold}

Descent of the submammary fold can occur if excessive surgical dissection is performed when the pocket is created because the patient presents cutaneous laxity or because the filler of the implant is inadequately cohesive (Fig. 5a-f). When the implant is fitted into a subglandular pocket, the submammary fold should be raised, and a capsulectomy should be performed in the upper area. If the envelope is lax, a retropectoral pocket should be created, and the implant should be replaced with one that is more cohesive.

When the implant is fitted submuscularly, the submammary fold should be raised. This involves performing a capsulectomy with excision of part of the capsule, which is anchored with permanent suture from the costal periosteum as far as the capsule, thus raising the groove (Fig. 6a), or it involves performing the "hammock technique" $[3,8]$ (Fig. 6b).

\section{Rippling}

Rippling may happen due to a lack of filling (in the vertical position, folds are produced) or because of traction. Generally, when the implant contains a large volume of liquid silicone, the lower pole expands further, increasing the distance from the areola to the submammary fold. The content is displaced downward, and the upper part of the implant is emptied, undulating the skin and producing rippling (Fig. 7).

This complication can be prevented as follows. If at the preoperative stage a pinch test is performed with results $<2 \mathrm{~cm}$, the implant should be fitted submuscularly. If the patient has substantial laxity, a cohesive gel implant with less volume should be fitted.

Rippling occurs with greater frequency when the implant is fitted subglandularly because in that case it is

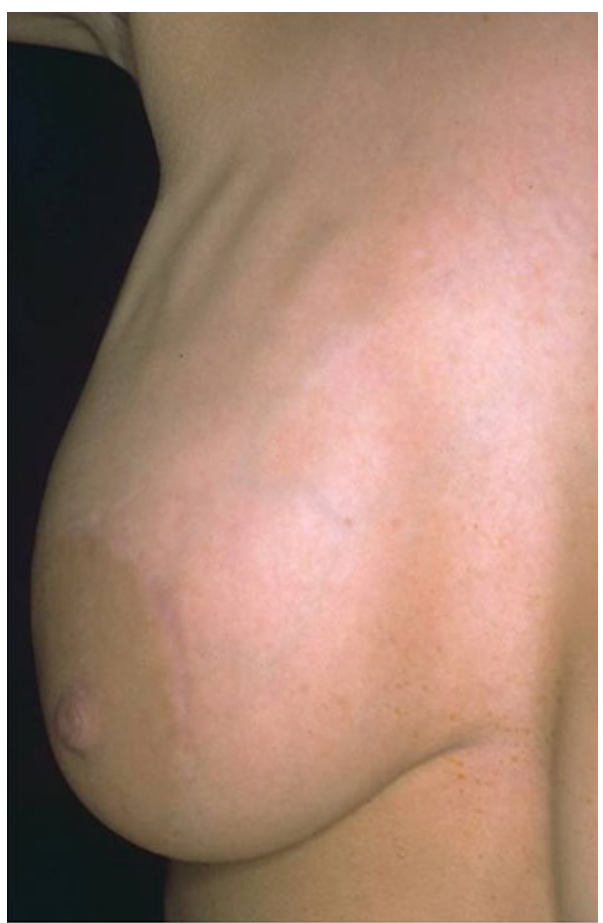

Fig. 7 Rippling in the upper pole

covered only by the envelope and the mammary gland, which in time becomes thinner and atrophied. Rarely does rippling occur when the implant is fitted submuscularly [3, $6,8]$.

When the implant is fitted in a subglandular pocket and the rippling is superior, the pocket should be changed, and this should be done submuscularly. If the rippling is medial, it is because the pocket is too close to the middle line. In this case, the pocket should be changed, with care taken to place it at least $1 \mathrm{~cm}$ lateral to the middle line $(0.5 \mathrm{~cm}$ of pectoral insertion) and to fit it submuscularly.

If the pocket is submuscular and the rippling is medial in the cleavage, generally this is because the pectoral muscle is disinserted too high. If this occurs, it should be lowered and sutured again in the middle line, and the pocket should be enlarged laterally. If rippling occurs because the implant
Fig. 6 Submammary fold descent. a Capsule suture. b Capsuloplasty hammock technique

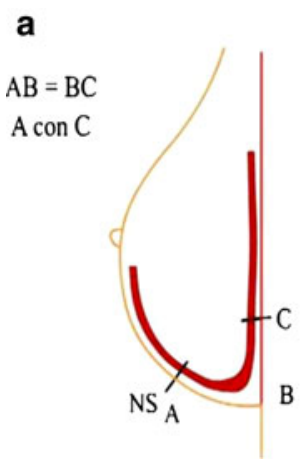

b

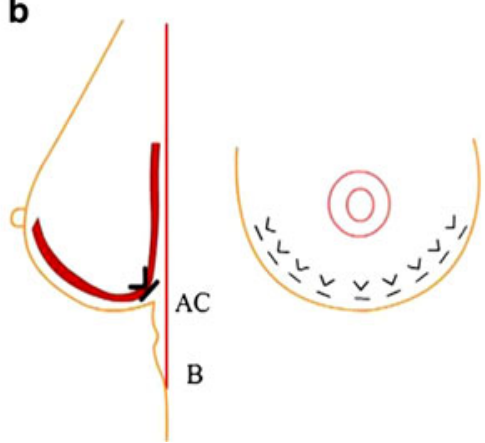

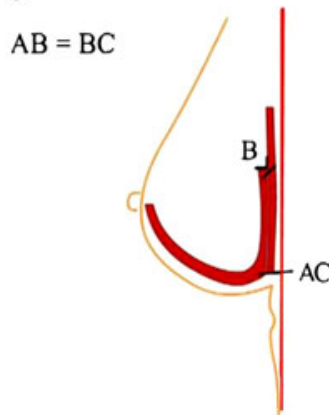




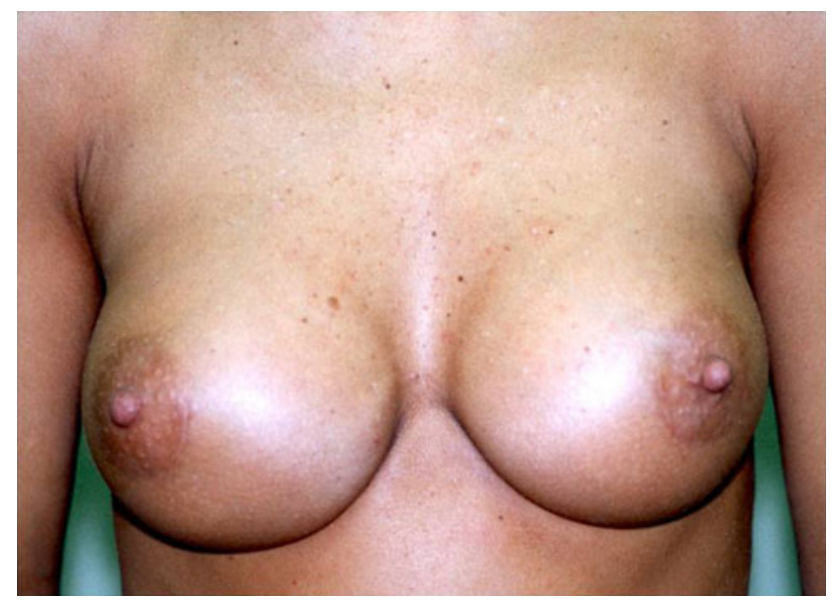

Fig. 8 Implant edge visible in the middle cleavage

is too large, it should be replaced with another smaller one. If it is a serum or gel implant, it should be replaced with a cohesive gel implant.

Fig. 9 a, c Double submammary fold. b, d The result. The surgery was performed through the lower edge of the areola

\section{Implant Visibility and Palpability}

Visibility and palpability of the implant can occur because the envelope is thin, because of excessive volume, because the implant's content is not cohesive, or because of previous surgery and cutaneous aging (Fig. 8).

If the implant is fitted in a subglandular pocket, a change to a submuscular pocket is needed. The volume of the implant should be reduced, and it should be ensured that the content is cohesive gel $[3,6,8]$.

\section{Double Submammary Fold}

Generally, a double submammary fold is produced when the implant is fitted submuscularly due to the insertion of the pectoral muscle into the tissues of the lateral envelope, with retraction of the capsule adhering to the muscular plane. For its correction, a capsulectomy should be performed, with all
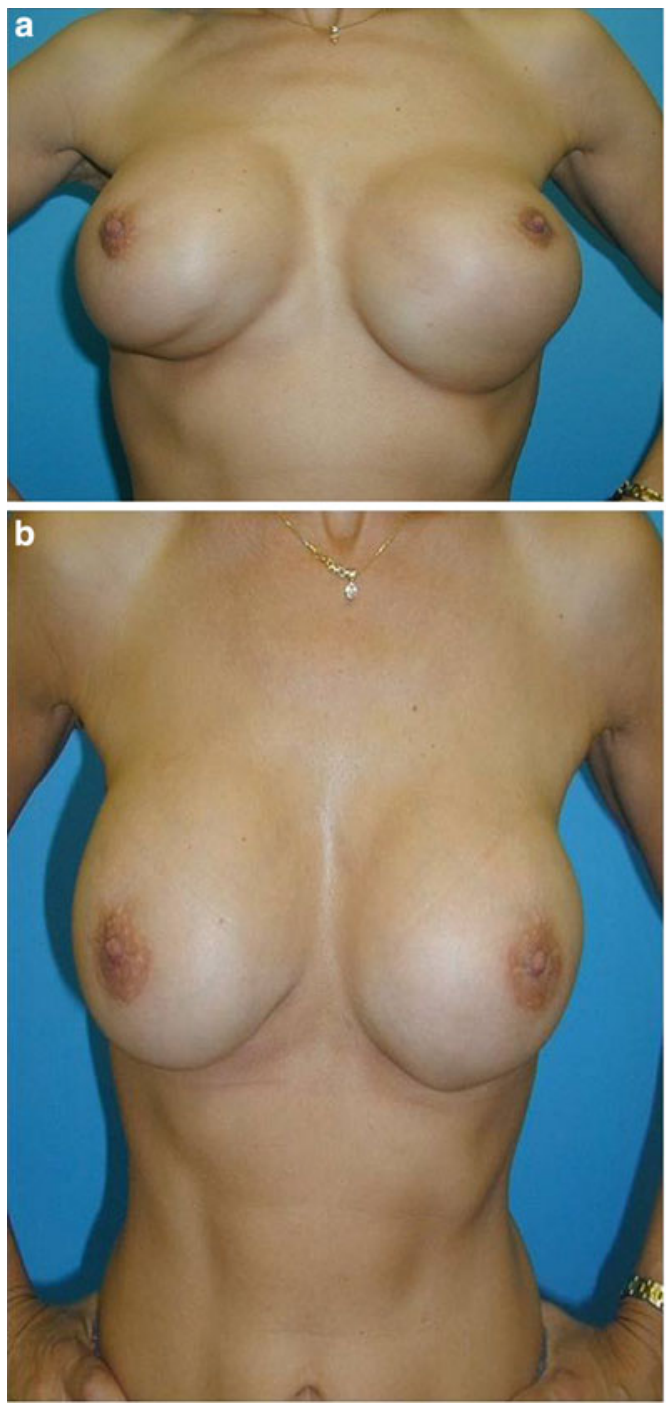

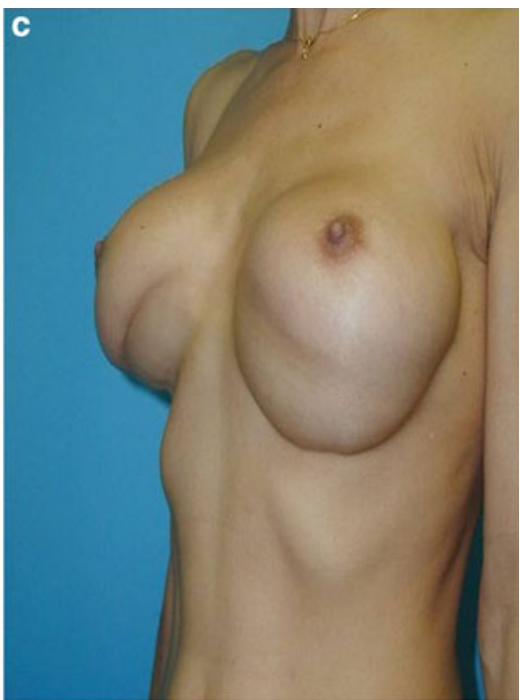

d

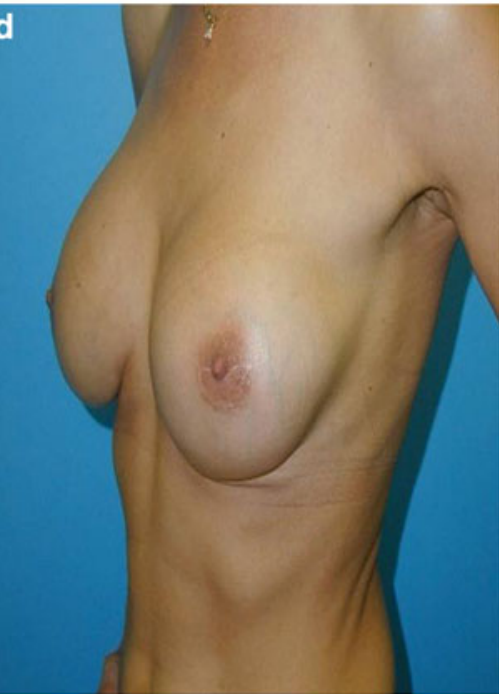


the tissues released as if this were the first operation. The tissue adherence of the pectoral muscle should be disinserted from the lateral cutaneous plane (Fig. 9a-d).

\section{Synmasty}

Synmasty is an infrequent complication that results from overdissection of the pocket in the medial region, from an excessively large implant, or from many previous breast operations. To correct synmasty, if the implant is fitted subglandularly, a capsulectomy should be performed, with the implant fitted in a submuscular pocket.

If the implant is fitted submuscularly, the pocket will close because it is very near the middle line, for which
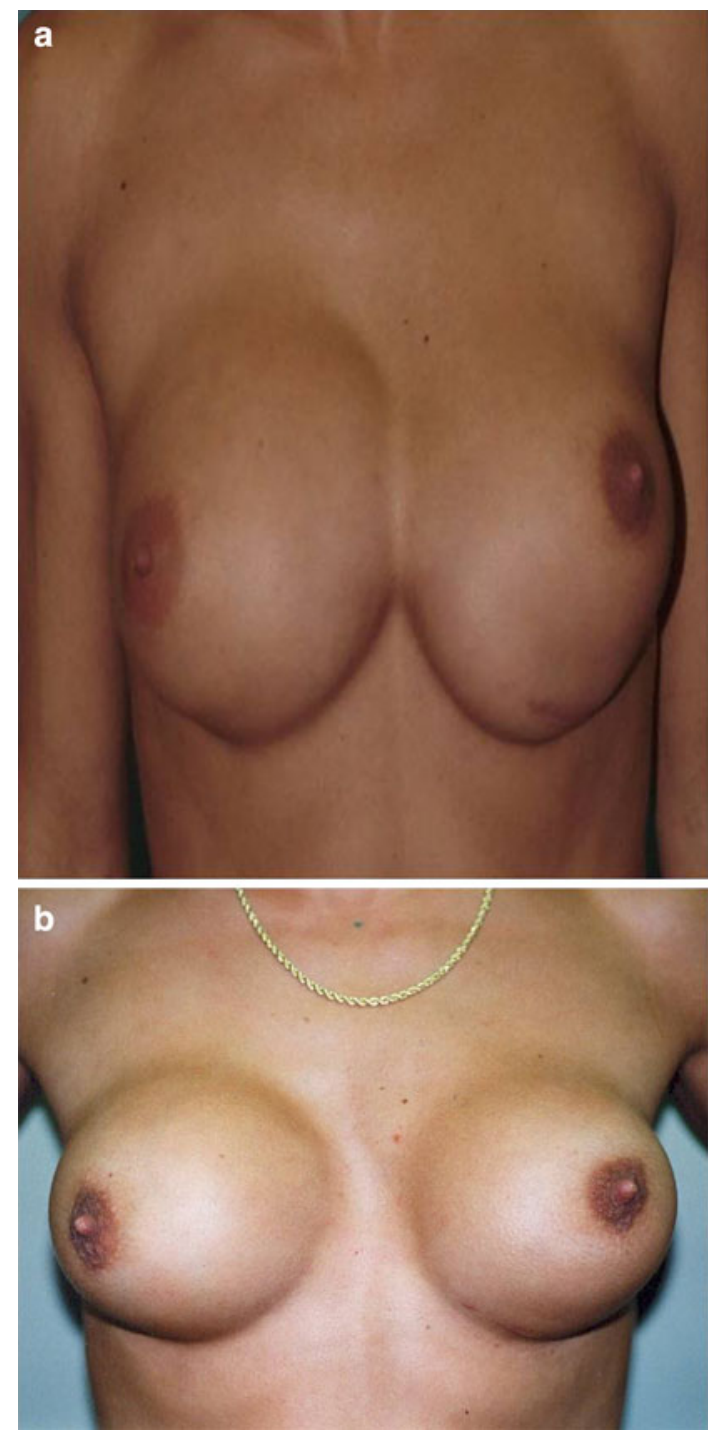

Fig. 10 a Synmasty. b The result. The middle cleavage distance is closed by capsuloplasty, lateral capsulotomy, and a narrow implant reason a capsulectomy is performed. The pectoral muscle should be sutured $1-2 \mathrm{~cm}$ lateral to the middle line, and an implant of less volume should be fitted (Fig. 10a and b).

\section{Implant Rotation}

Rotation of the implant can occur due to an error made choosing the implant in relation to the pocket, excessive lateral dissection of the pocket, a lax envelope, the presence of a seroma or hematoma, or an inadequate relation between the size of the pocket and the implant. When the implant is large, it has more weight and greater expansion, with the result that a fall and rotation of the implant may take place.

The solution to this complication when the implant is fitted into a subglandular pocket is to perform a capsulectomy and to create a submuscular pocket for the new implant. If the implant is fitted submuscularly, a lateral pocket should be chosen (Lelouan's technique). In both cases, the anatomic implant should be replaced with a
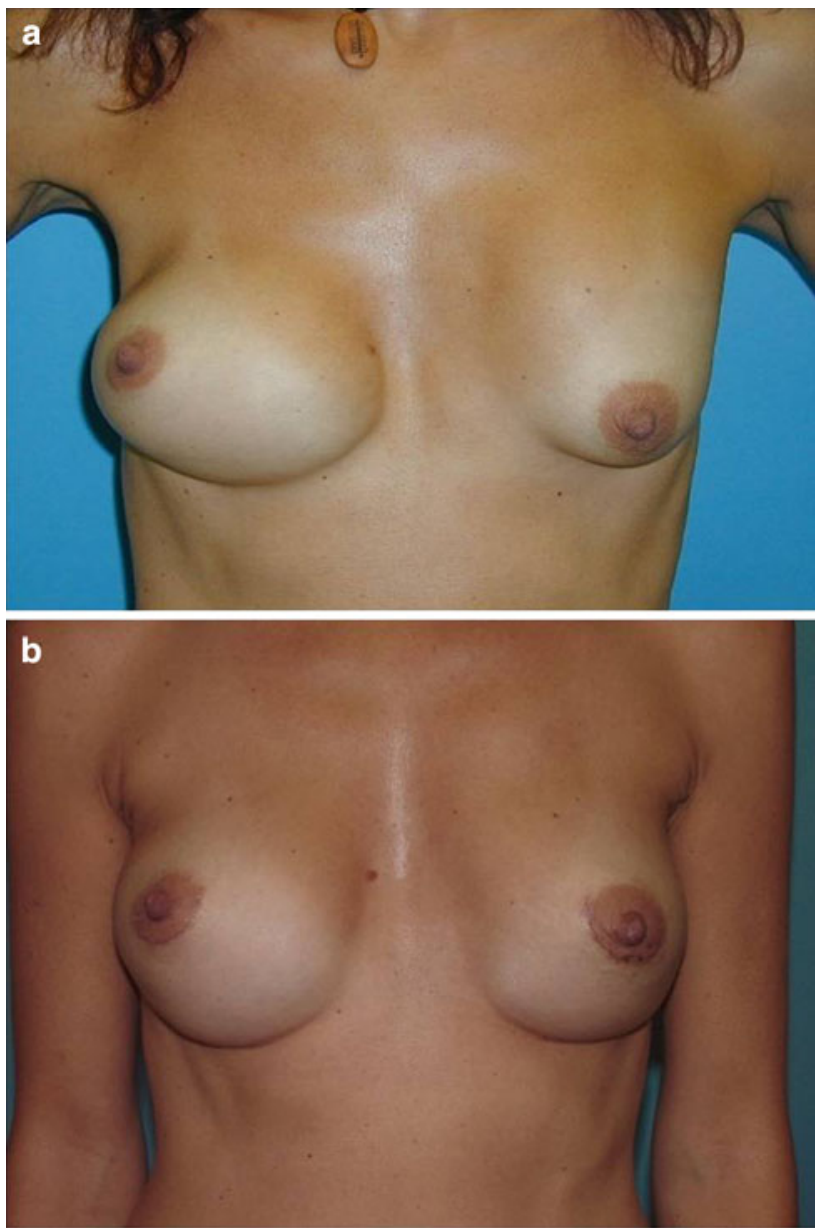

Fig. 11 a Implant rotation in the right breast. b The result 
round implant. When the envelope is lax, a round submuscular implant should be fitted immediately to avoid implant rotation [5] (Fig. 11a and b).

\section{Implant Infection or Extrusion}

Infection or extrusion of the implant can be associated with failure to perform adequate aseptic methods, with contamination caused by mammary secretions proceeding from the mammary ducts, and with the use of drainage, which creates communication with the skin and the exterior. The symptoms are redness, pain, increased temperature, and hardening of the breast, among others.

To prevent this complication, great care should be taken to use aseptic methods, to irrigate the pocket with Povidone, and to prescribe intra- and postoperative antibiotics. Once the infection manifests itself, samples must be taken for culture and antibiogram. If the infection is acute, the implant must be removed, and the whole area must be washed with Povidone and a general antibiotic. Only after a 3-month wait should another implant be inserted once the infection has resolved and the normal consistency of the adjacent tissues has been recovered [3, 6] (Fig. 12).

\section{Implant Rupture}

Rupture of the implant generally occurs in less than $1 \%$ of cases. The rupture can be intra- or extracapsular (Fig. 13a and b). It can be caused by various factors including defects in the manufacture of the implant or deterioration, the passing of time, the shell of the implant, trauma, or an unnoticed rupture during surgery.

Rupture of the implant is associated with the breast's loss of shape. Physical examination can determine the diagnosis.

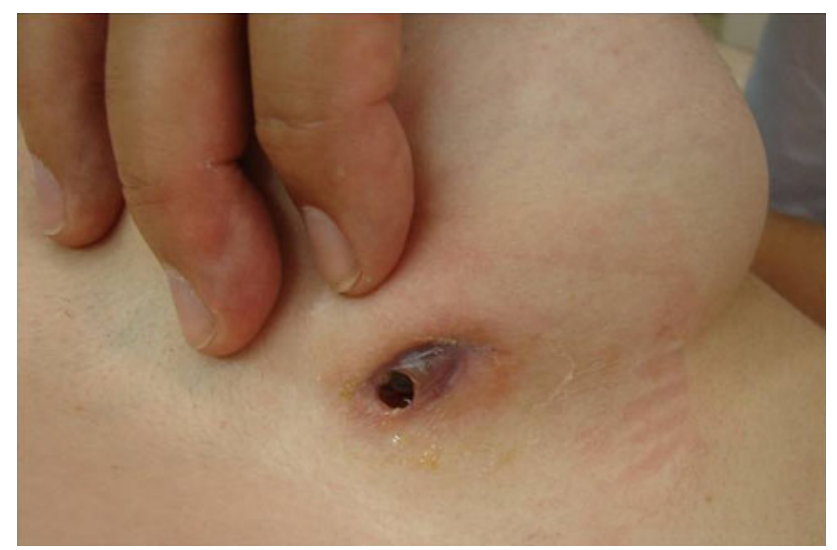

Fig. 12 Infection and extrusion
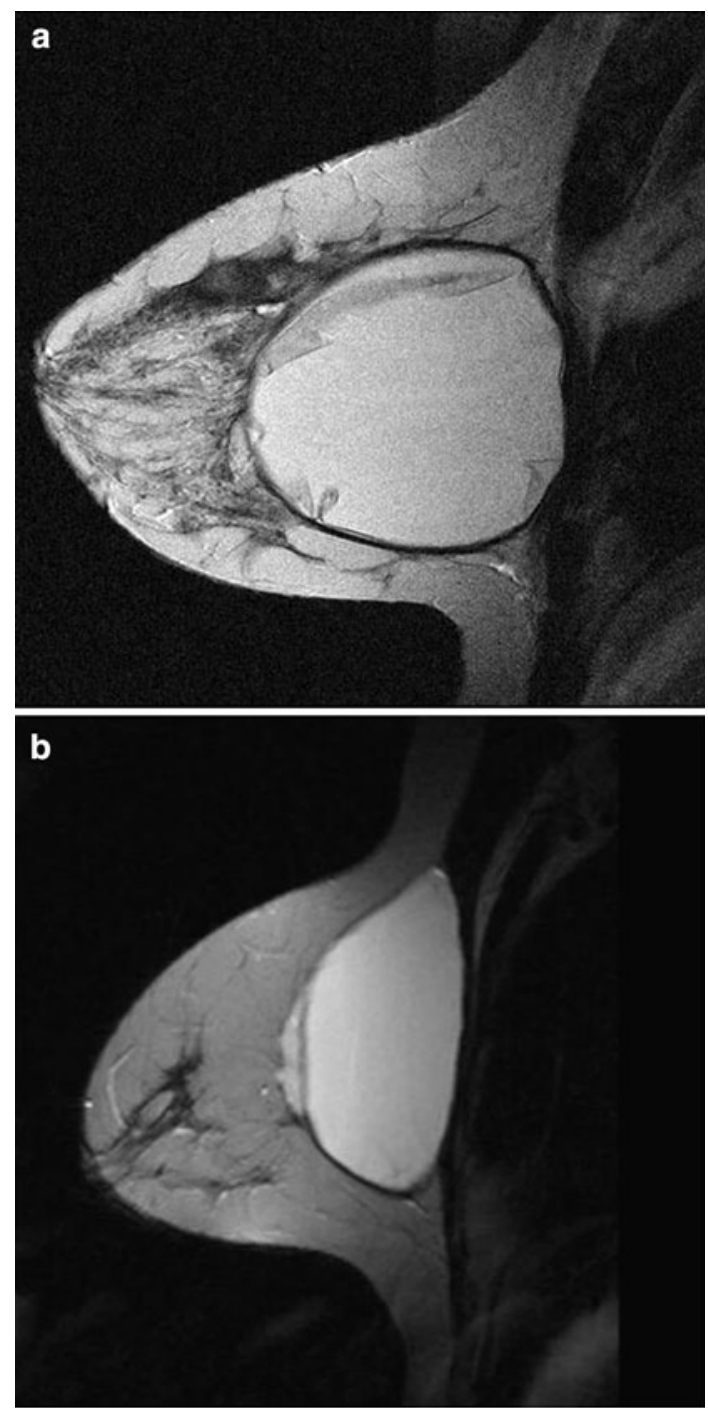

Fig. 13 a Magnetic resonance image (MRI) of intracapsular rupture (Linguine sign). b An MRI of extracapsular rupture

The breast can be touched when it is extracapsular but not when it is intracapsular. However, because a margin of error is always possible, the diagnosis should be corroborated by a magnetic resonance imaging (MRI) study.

When cohesive silicone implants rupture, the principal symptoms are contour irregularity and a palpable mass attributable to the capsular contracture. The symptoms of a ruptured saline implant are deformity of the contour and an abrupt collapse. With the rupture of liquid silicone gel implants, the symptoms are deformity of the contour, redness, and pain.

Inflatable saline implants can become deflated due to valve failure or leakage through folds, which happens when the implant becomes folded at some point, thus producing friction between the edges of the folds until the shell is ruptured. Should this occur, the implant must be extracted 
and the pocket cleaned, followed by the fitting of a new cohesive gel implant [9].

\section{Asymmetry}

Preoperative asymmetries include areolas in different positions medially or with regard to height, different breast shapes (e.g., one round and the other tuberous), or different breast sizes. It is essential to inform the patient about all these details, which are well documented, and to discuss with the patient the various therapeutic options. These types of asymmetry should be differentiated from a postoperative aesthetic difference in the two breasts produced by factors described previously such as a fall of the fold, a high implant, or a rotation of the implant [5].

Asymmetries caused by the unequal fitting of the implants or by the creation of different submammary grooves are a result of technical values and can be prevented by means of adequate preoperative planning, correct dissection of the pockets, and comparison of the two breasts after fitting of the implants. It is possible that after a breast augmentation, small deformities in the wall of the
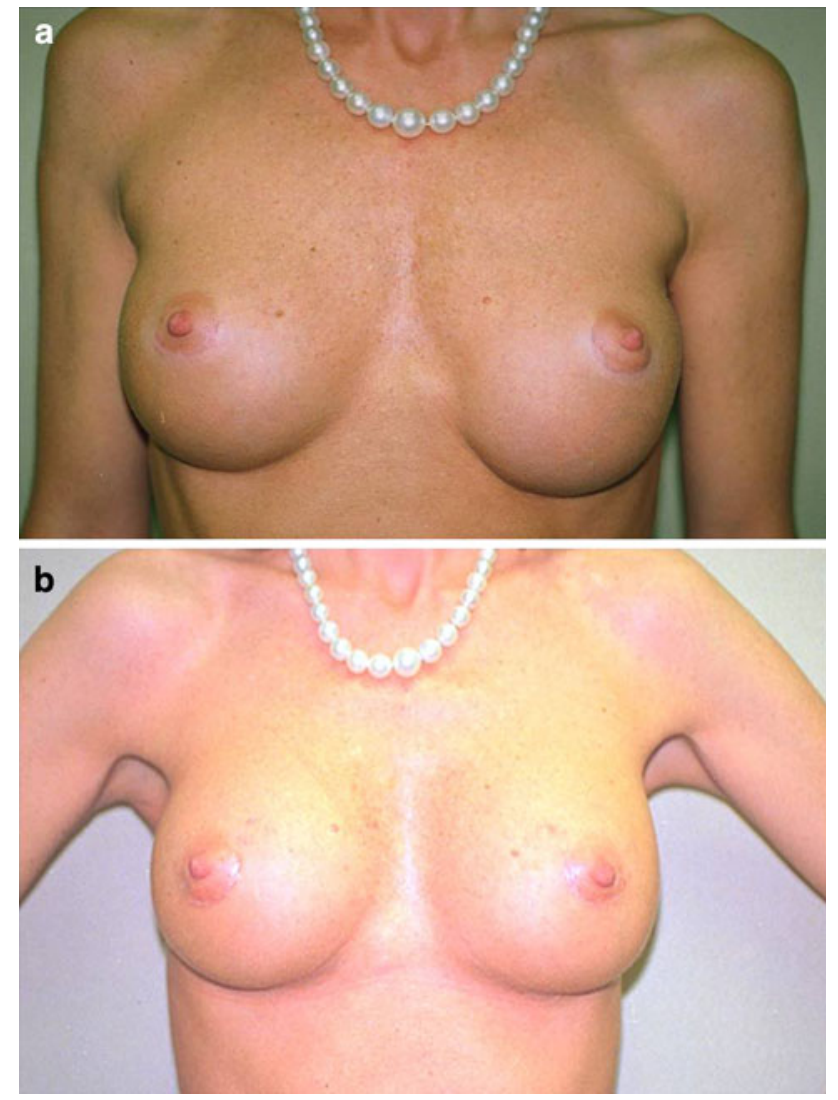

Fig. 14 a Left fold retraction and subglandular implant descent. Regular gel filler. b Submammary fold correction, new subpectoral pocket, and cohesive gel filler thorax or a morphologic mammary disorder may become much more evident. For this reason, the predicted correction of these anomalies should be discussed with the patient before her operation (Fig. 14a and b).

\section{Changes in Breast Volume Caused by the Presence of Liquid}

A change in breast volume can be caused by a hematoma or seroma. If the change occurs immediately, within 7 days of
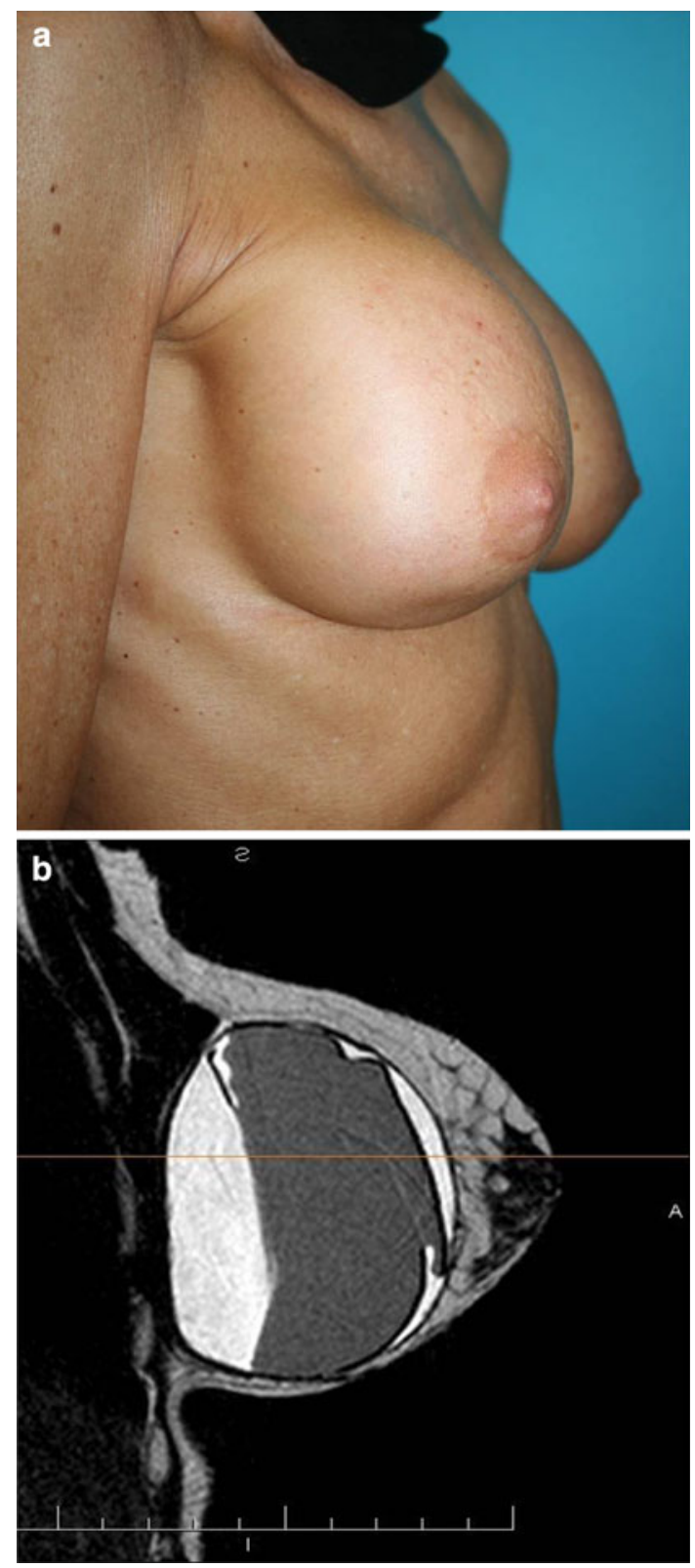

Fig. 15 a Breast volume increased by liquid 16 years after breast augmentation. b Magnetic resonance image (MRI). Almost all the seroma is located behind the implant 
surgery, it generally is due to a hematoma and will manifest as pain, hardening of the breast that does not diminish in a few days, and an increase in volume. If the change in volume is immediate and large, drainage should be performed within the first $24 \mathrm{~h}$. Treatment with anti-inflammatory drugs, antibiotics, and ultrasound should be administered within a week to stimulate reabsorption.

Although rare, delayed hematoma can appear after weeks, months, or even years. It may be due to secondary bleeding or microfractures in the capsule induced by microtrauma, microinflammation, or an increase in capillary permeability. The capsule formed around the implant serves as a barrier that can limit the hematoma, thus preventing contamination. A delayed hematoma should be evacuated as soon as it is diagnosed. This evacuation may coincide with a closed capsulectomy.

If the increase in volume occurs months or years later (Fig. 15a and b), it almost always is the result of a seroma. Such a volume increase is soft to the touch, which distinguishes it from a hematoma, which is hard. The treatment should include antiinflammatory drugs, diuretics, initial observation, and suction drainage (Fig. 16). The differential diagnosis between hematoma and seroma should be performed with an MRI when the clinical diagnosis is not sufficient.

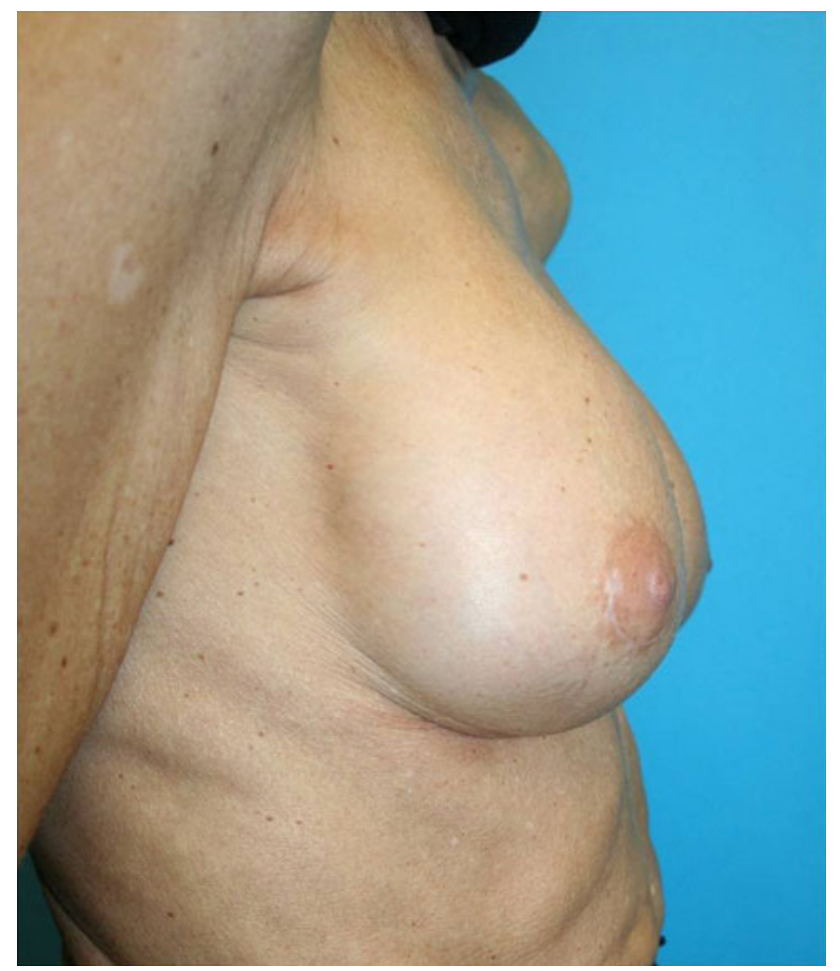

Fig. 16 Result after removal of the liquid by drainage

\section{Changes in Volume Requested by the Patient}

If the patient wants an increase in breast volume and the implant is subglandular, a lateral capsulotomy with enlargement of the pocket can be performed and a larger implant fitted. If the pocket is submuscular, the lateral capsulotomy can be performed to enlarge the pocket. Then an implant bigger can be fitted.

If the patient wants a reduction in breast volume and the implant is subglandular and round, the implant can be replaced with a smaller one if the same pocket is retained. However, if the implant is anatomic, the pocket can be closed and the implant replaced with a round implant in a submuscular pocket. When the implant is fitted submuscularly, the pocket can be closed with capsuloplasty and sutures after insertion of a smaller implant (Fig. 17a and b).

\section{Changes Caused by Aging}

Patients may notice changes in their breasts due to significant involution caused by pregnancy, lactation, menopause, and changes in weight or lifestyle. Mammary ptosis
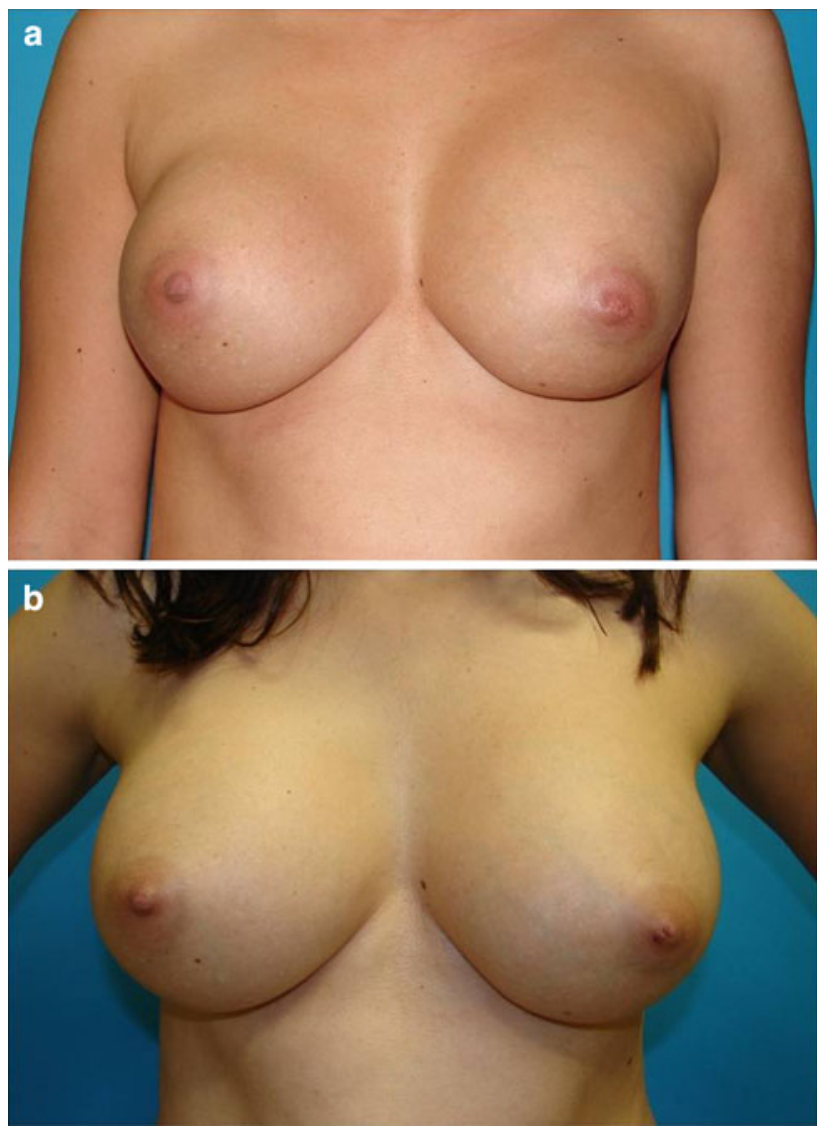

Fig. 17 a The patient wishes to increase the implant volume. b The result 
is a disorder associated with the aging process that results from glandular atrophy, loss of cutaneous elasticity, or weakening of ligament structures of the mammary support. Ptosis worsens with pregnancy and lactation due to the associated hypertrophy and subsequent atrophy. The global effect of these changes results in an unaesthetic fall of the breasts.

\section{Conclusion}

It is important that surgeons have knowledge of the options available to treat a breast augmentation problem. They must bear in mind that as the number of breast operations increases, the guarantees become fewer that the breast can be improved.

Open Access This article is distributed under the terms of the Creative Commons Attribution Noncommercial License which permits any noncommercial use, distribution, and reproduction in any medium, provided the original author(s) and source are credited.

\section{References}

1. Young VL, Waston ME (2001) Breast implant research. Where we have been, where we are, where we need to go. Clin Plast Surg $28: 451-452$
2. Conder MA, Cohen AT, Hester TR (2001) Complications in breast augmentation: prevention and correction. Clin Plast Surg 28:587595

3. Wiener TC (2008) Relationship of incision choice to capsular contracture. Aesth Plast Surg 32:303-306

4. Tebbetts JB (2001) Patient evaluation, operative planning, and surgical techniques to increase control reduce morbility and reoperations in breast augmentation. Clin Plast Surg 28:501-521

5. Aranco A, Gravante G, Araco F et al (2007) A retrospective analysis of 3, 000 primary aesthetic breast augmentations: postoperative complications and associated factors. Aesth Plast Surg 31:532-539

6. Omar D, Ventura H, Gaspar A (2008) Treatment of the fibrous capsule on mammary implants: a technical innovation for changing the implant from the subglandular to the submuscular plane. Aesth Plast Surg 32:365-367

7. Tebbets JB (2001) Breast augmentation with full-height anatomic saline implants. The pros and cons. Clin Plast Surg 28:567-577

8. Spear S, Carter M, Ganz J (2006) The correction of capsular contracture by conversion to dual-plane positioning: technique and outcomes. Plast Reconst Surg 118:103-113

9. Tark KC, Jeong HS, Roh TS (2005) Analysis of 30 breast implant rupture cases. Aesth Plast Surg 29:460-469 